\title{
A new approach for flexible protein-ligand docking based on Particle Swarm Optimisation Rene Meier*1, Frank Brandt' ${ }^{2}$, Teresa M Pisabarro ${ }^{2}$, Carsten Baldauf ${ }^{2}$ and Wolfgang Sippl ${ }^{1}$
}

\author{
Address: ${ }^{1}$ Institute for Pharmaceutical Chemistry, Martin-Luther-University Halle-Wittenberg Wolfgang-Langenbeckstr. 4, 06120 Halle (Saale), \\ Germany and 2Structural Bioinformatics, Biotec TU Dresden, Tatzberg 47-51, 01307 Dresden, Germany \\ Email: Rene Meier* - rene.meier@pharmazie.uni-halle.de \\ * Corresponding author
}

from 3rd German Conference on Chemoinformatics

Goslar, Germany. II-I3 November 2007

Published: 26 March 2008

Chemistry Central Journal 2008, 2(Suppl I):P6 doi:10.1186/I752-I53X-2-SI-P6

This abstract is available from: http://www.journal.chemistrycentral.com/content/2/SI/P6

(c) 2008 Meier et al.

Particle Swarm Optimiser (PSO) uses a general-purpose, iterative, heuristic search algorithm. It considers a population of individuals to probe promising regions of the search space in an effective manner. In this context, the population of solutions is called a swarm, and the individuals are called particles. Each particle moves within the search space and retains in its memory the best position and the overall best position that has been encountered. The velocity of each particle is adjusted during each iteration toward the personal best position as well as the overall best position, thus mimicking swarm intelligence. In our recent work we have implemented PSO in a liganddocking program. The fitness landscape of the docking program is modeled by a modified version of the scoring function X-Score [1]. X-Score is an empirical scoring function which shows a significant correlation between calculated docking scores and experimentally derived ligand geometries. Preliminary investigations show promising results in terms of speed and accuracy. Special attention during the development will be paid to a modular design of the program in order to easily implement different scoring functions as well as to perform parallel computing.

\section{References}

I. Wang R, Lai L, Wang S: J Comput-Aided Mol Des 2002, 16:I I-26. 